\title{
Deforestación en ecosistemas templados de la precordillera andina del centro-sur de Chile
}

\author{
Deforestation in temperate ecosystems of pre-Andean range of south-central Chile
}

\author{
Adison Altamirano ${ }^{a^{*}}$, Antonio Lara ${ }^{b}$ \\ *Autor de correspondencia: aUniversidad de La Frontera, Departamento de Ciencias Forestales, casilla 54-D, Temuco, Chile, \\ tel.: 56-45-734159, aaltamirano@ufro.cl \\ bUniversidad Austral de Chile, Instituto de Silvicultura, Valdivia, Chile.
}

\begin{abstract}
SUMMARY
A multitemporal analysis to determine the land cover change was conduced in the pre-Andean range of Maule region of Chile. We used satellite imagery from the year 1989 and 2003 to analyze the likely causes of native forest change. In this period, there was a reduction in native forest area of $44 \%$ in the study area, which is equivalent to an annual forest loss rate of $4.1 \%$. The native forest area is replaced by shrublands (29\%) and exotic forest plantations (27\%). A great proportion of current exotic forest plantations are established on lands that were native forest in 1989 (63\%). Most forest loss was associated with intermediate elevation and less distance to road network, where native forest area dominated. Native forest replacing by shrublands was associated to selective forest logging and firewood, which leads to degradation of these forests. Native forest replacing by exotic forest plantations was the result of the forestation incentive and an increase in the demand for forest products such as wood and pulp. Because of multiple impacts of the forest loss, we propose to focus the government incentives. Priority areas for incentives must be characterized by high forest loss rate and poverty, presence of threatened species and low availability of water from catchments. Moreover, we propose to prioritize the connectivity of the current network protected areas and to develop an effective forest management monitoring system using advanced technologies.
\end{abstract}

Key words: Maule region, native forest, multitemporal analysis, remote sensing, land cover.

\section{RESUMEN}

Se realizó un análisis multitemporal para determinar los cambios en la cobertura del suelo en una zona de la precordillera andina de la Región del Maule, Chile. Para ello se utilizaron imágenes satelitales de los años 1989 y 2003, y se analizaron las causas probables de la deforestación del bosque nativo presente en estos ecosistemas. El bosque nativo se redujo en un $44 \%$, lo cual se traduce en una tasa de deforestación de 4,1\% anual. La superficie de bosque nativo fue reemplazada preferentemente por coberturas de matorral (29\%) y plantaciones de especies exóticas (27\%). Una gran proporción de las actuales plantaciones exóticas está establecida sobre terrenos que anteriormente correspondían a bosque nativo (63\%). La mayor pérdida de bosque nativo se concentra en niveles de elevación intermedia, donde se encuentra la mayor superficie de bosque nativo, asociándose también a una menor distancia de la red de caminos. El reemplazo del bosque nativo por matorrales se asocia principalmente a prácticas de floreo y extracción de leña, lo cual produce la degradación del bosque. La sustitución de bosque nativo por especies exóticas se explica por el incentivo a la forestación y por la creciente demanda de productos derivados de la madera y pulpa. Debido a los múltiples impactos que produce la deforestación se propone focalizar los incentivos estatales en áreas con altos niveles de deforestación y pobreza, presencia de especies amenazadas y menor disponibilidad de agua desde las cuencas. Adicionalmente, se propone priorizar la conectividad de las actuales áreas protegidas y el desarrollo de un sistema de fiscalización eficaz para el manejo de los bosques, el cual considere un monitoreo permanente con uso de tecnologías avanzadas.

Palabras clave: Región del Maule, bosque nativo, análisis multitemporal, sensores remotos, cobertura del suelo.

\section{INTRODUCCIÓN}

Los ecosistemas se encuentran sometidos a cambios constantes, los cuales se producen a diferentes escalas espaciales y temporales (Coppin et al. 2004). Uno de los principales cambios que los afectan actualmente es la deforestación, la cual tiene como principal causa la intervención antrópica (Alves 2002, Cayuela et al. 2006, Echeverría et al. 2006). La reducción de los bosques y el cambio de uso del suelo juegan un papel clave en el control del calentamiento global, debido a que secuestran las emisiones de carbono desde la atmósfera y a la vez son un reservorio de éste a largo plazo (Sheeran 2006). Al mismo tiempo, se ha reportado que la deforestación 
afecta al ciclo hidrológico, reduciendo la evapotranspiración y produciendo un aumento de los caudales (Sun et al. 2005, D'Almeida et al. 2006). El empobrecimiento de los suelos, producto de la erosión, también se asocia a la pérdida de cobertura forestal, principalmente porque la ausencia del bosque aumenta la escorrentía superficial del agua (Mainville et al. 2006).

La cuantificación, determinación de causas y efectos de la deforestación ha sido ampliamente reportada para ecosistemas tropicales, especialmente en regiones de la Amazonía (Laurance et al. 2002, de Barros et al. 2005, Armenteras et al. 2006, D'Almeida et al. 2006, Etter et al. 2006, Zimmermann et al. 2006). Sin embargo, en los bosques templados de Chile es aún incipiente la investigación que contribuye a explicar este proceso (Lara et al. 1989, Bustamante y Castor 1998, Olivares 1999, Echeverría et al. 2006, 2008).

En términos generales, los últimos estudios señalan que los bosques templados de Chile están siendo cosechados para suplir la creciente demanda internacional de productos derivados de la madera y el papel, así como para la habilitación de áreas para cultivos agrícolas y praderas (Lara et al. 2002, Echeverría et al. 2006, 2008). La intensidad de los cambios, así como las probables causas varían dependiendo de las zonas estudiadas. Esta situación mantiene en una constante amenaza a estos ecosistemas, que se consideran verdaderas islas biogeográficas debido al aislamiento al cual han estado sometidos en períodos glaciales y las barreras naturales actuales como desiertos, cordilleras y océanos (Donoso 1994, Armesto et al. 1998). Los bosques templados de Chile son considerados como una de las áreas más importantes de la biodiversidad a nivel mundial (Myers et al. 2000), ello debido principalmente a los altos niveles de endemismo y géneros monotípicos (Armesto et al. 1998). Además, la Iniciativa Global 200 de WWF y el Banco Mundial han incluido a esta zona como una de las ecorregiones más amenazadas del mundo (Dinerstein et al. 1995).

Algunas de estas zonas poseen ciertas particularidades, como lo son las áreas precordilleranas, las cuales además han sido escasamente estudiadas. Por ejemplo, algunos estudios han medido la pérdida de cobertura de bosque nativo en áreas precordilleranas de la Región del Maule de Chile (Lara et al. 1989, Olivares 1999), aunque éstos presentan algunas limitaciones respecto al uso de información con diferente escala y otras tecnologías como fotografías aéreas, lo cual limita las estimaciones y comparaciones con estudios que utilizan otro tipo de metodologías (Kerr y Ostrovsky 2003, Aplin 2004). Estas áreas revisten una clara importancia ya que albergan muchas especies endémicas, estando algunas de ellas en condición de amenazadas como: Austrocedrus chilensis (D. Don.) Pic.Serm. et Biz., Beilschmiedia berteroana (Gay) Kosterm., Cytronella mucronata (Ruiz et Pavón) D. Don., Legrandia concinna (Philippi) Kausel. y Nothofagus glauca (Phil.) Krasser. (Hechenleitner et al. 2005). Por otro lado, en estas áreas se concentra gran parte de la producción hidroeléctrica del país y se ha manifestado un aumento de la actividad forestal asociada al cultivo de plantaciones de especies exóticas (Lara et al. 2003). Estas actividades producen un fuerte impacto sobre el uso del suelo, y hacen pronosticar una pérdida substancial de bosque nativo en los últimos años. Ante esto, es necesario conocer los patrones de cambio en estos ecosistemas, evaluar su grado de amenaza y proponer medidas que mitiguen los impactos negativos que pueden producir estos cambios. En el presente estudio se determinaron los cambios en la cobertura del suelo entre los años 1989 y 2003 en ecosistemas precordilleranos de la Región del Maule de Chile. En particular, se analizaron las probables causas del cambio del bosque nativo presente en estos ecosistemas. A partir de estos resultados se proponen medidas que ayuden a disminuir los actuales niveles de deforestación y mitigar los efectos que pueden producir estos cambios.

\section{MÉTODOS}

Área de estudio. La delimitación del área de estudio consideró el sector pre-andino de la Región del Maule de Chile, abarcando la mayor parte de las comunas que componen este sector: San Clemente, Colbún, Linares y Longaví. Esta delimitación también consideró la eliminación de sectores orientales donde el desarrollo de la vegetación es limitado debido a las condiciones ambientales. Esta área se ubica aproximadamente entre $\operatorname{los} 35^{\circ}$ y $36^{\circ}$ de latitud sur. La superficie aproximada es de 170.000 ha (figura 1) y varía en rangos de altitud entre 200 y 2.000 m s.n.m. Los suelos predominantes son de origen volcánico con diferente grado de desarrollo (Schlatter et al. 1997). El clima predominante corresponde al tipo mediterráneo (di Castri y Hajek 1976), caracterizado por precipitaciones entre aproximadamente 1.500 y $2.100 \mathrm{~mm}$ anuales concentradas en el invierno, y temperatura media anual entre 11,8 y $13^{\circ} \mathrm{C}$ (Pezoa 2003).

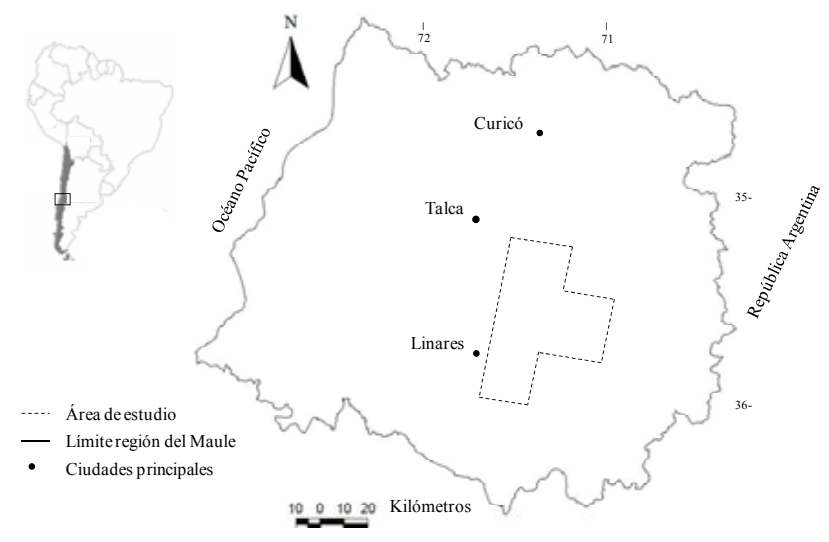

Figura 1. Mapa del área de estudio.

Map of the study area. 
El área de estudio es altamente representativa de la región debido a la composición de los bosques. Los bosques nativos en esta zona precordillerana son caracterizados por la presencia de las especies arbóreas Nothofagus obliqua, $N$. glauca, N. dombeyi, Criptocarya alba, Quillaja saponaria y Lithraea caustica, además de plantaciones industriales de especies forestales y bosques mixtos de especies nativas y exóticas (CONAF et al. 1999). A su vez, esta área es representativa de condiciones más generales del bosque templado de la ecorregión valdiviana de Chile sur-central bajo un clima templado con influencia mediterránea.

El establecimiento de colonos europeos a mediados del siglo XX en esta región produjo una alta pérdida de bosque nativo debido a la habilitación de tierras destinadas principalmente a la agricultura (Donoso y Lara 1995). En los últimos años la intervención antrópica en estos bosques se ha caracterizado por la corta de árboles en pequeñas superficies para prácticas agrícolas de subsistencia y la extracción de madera para construcción y material combustible (Olivares 1999). Ello ha producido una continua degradación del bosque y un empobrecimiento del suelo, dando origen a formaciones de matorrales bajos dominados por especies esclerófilas (Donoso 1994, San Martín y Donoso 1995).

Información satelital y procesamiento de imágenes satelitales. Fueron utilizadas dos imágenes satelitales, una del año 2003 y otra del año 1989. La imagen del año 2003 correspondió a una escena Aster de fecha 24 de marzo y la del año 1989 correspondió a una imagen Landsat TM de fecha 17 de marzo. Debido a que la imagen Aster posee una resolución espacial (tamaño de píxel) de $15 \mathrm{~m}$ y la imagen Landsat de $30 \mathrm{~m}$ se aplicó un procedimiento de resampling, dejando a ambas imágenes con una resolución espacial de $30 \mathrm{~m}$, para poder realizar comparaciones cuantitativas.

Cada imagen fue georreferenciada a partir de, aproximadamente, 40 puntos de control obtenidos de coberturas vectoriales de caminos y ríos provenientes de bases de datos del catastro de la vegetación nativa (CONAF et al. 1999), alcanzándose en ambas imágenes un error menor a un píxel (Lillesand et al. 2004). Posteriormente, cada escena fue corregida atmosféricamente mediante el método de substracción de píxeles oscuros, utilizando objetos como cuerpos de agua (Mather 1999). Áreas con topografía muy abrupta presentan dificultades para la posterior clasificación de la imagen satelital debido a cambios en los valores espectrales. Sin embargo, estas áreas representaron un porcentaje menor a $0,3 \%$ del área de estudio, debido a lo cual fueron excluidas del análisis (Baldyga et al. 2007, Broadbent et al. 2008). Todo este proceso se realizó con el software ERDAS Imagine 8.4®.

Clasificación y validación de imágenes satelitales. Se aplicó un procedimiento de clasificación supervisada a ambas imágenes, en el cual se establecieron áreas de entrenamiento para cada clase de cobertura del suelo (Lillesand et al. 2004). Cada clase se estableció utilizando más de 200 píxeles por cada una como áreas de entrenamiento. Las clases de cobertura del suelo se identificaron utilizando diferentes fuentes de información, como mapas de vegetación y fotografías aéreas del año $1986^{1}$, fotografías aéreas del año $2003^{2}$ y visitas a terreno efectuadas entre los años 2004 y 2006. Estas clases son detalladas en la siguiente sección.

Cada imagen se clasificó en etapas, permitiendo agregar información auxiliar como análisis de textura y componentes principales para mejorar la exactitud de la clasificación (Lillesand et al. 2004). La imagen Aster del año 2003 se clasificó en una primera etapa utilizando las bandas espectrales 1,2 y 3 (correspondientes a las zonas del verde, rojo e infrarrojo cercano en el espectro electromagnético, respectivamente). Con las mismas áreas de entrenamiento se clasificó nuevamente la imagen agregando una nueva banda de textura a las bandas espectrales 1,2 y 3 . La banda de textura fue creada usando las bandas 1,2 y 3, y expresa la similitud espectral entre píxeles adyacentes en ventanas de 5 x 5 píxeles (Lillesand et al. 2004). Un último procedimiento de clasificación fue aplicado agregando a las bandas anteriores una banda generada mediante un análisis de componentes principales que resume la información de las tres primeras bandas espectrales de la imagen.

La imagen Landsat del año 1989 se clasificó inicialmente utilizando las bandas espectrales 1, 2, 3 y 4 (correspondientes a las zonas del azul, verde, rojo e infrarrojo cercano del espectro, respectivamente). Con las mismas áreas de entrenamiento se clasificó nuevamente la imagen agregando la banda 5 (infrarrojo medio) y posteriormente la banda 6 (infrarrojo medio). Ambas escenas fueron clasificadas utilizando el software ERDAS Imagine 8.4®, aplicando el método de clasificación de máxima verosimilitud (Lillesand et al. 2004).

Para evaluar las áreas de entrenamiento se utilizó el índice de divergencia transformado, el cual varía entre 0 y 2.000 , indicando la mínima y máxima separabilidad espectral entre pares de clases de cobertura del suelo, respectivamente (ERDAS 1999). Posteriormente se evaluó la exactitud de la clasificación supervisada a través de la selección de un mínimo de 50 píxeles (muestras independientes de las áreas de entrenamiento) por cada clase de cobertura del suelo en las imágenes clasificadas, y luego verificados mediante visitas a terreno e información del área de estudio. Con esta información se construyó una matriz de errores.

\footnotetext{
Mapas de vegetación a escala 1: 50.000 elaborados por Antonio Lara para la empresa ENDESA en 1985, utilizando fotografías aéreas. Fotografías aéreas facilitadas por CONAMA VII Región.
} 
Cambios en la cobertura del suelo y el bosque nativo. En ambas imágenes satelitales se reconocieron las siguientes clases de cobertura del suelo: terrenos agrícolas, plantaciones forestales (principalmente de Pinus radiata D. Don); matorral que incluye las subcategorías matorral denso y matorral abierto, compuestos principalmente por especies esclerófilas de menor altura; matorral arborescente (situación intermedia entre matorral y renoval) ${ }^{3}$; bosque nativo que incluye las subcategorías bosque de Nothofagus spp. (principalmente renovales y también bosque adulto y renoval dominado por especies como Nothofagus obliqua (Mirb.) Oerst. y $N$. glauca) y renoval de especies esclerófilas sobre $2 \mathrm{~m}$ de altura y $>50 \%$ de cobertura con especies como Criptocarya alba (Mol.) Looser., Quillaja saponaria Mol. y Lithraea caustica Hook. et Arn.; pastizales (principalmente praderas de uso ganadero y esteparias), suelos desnudos, nieve y cuerpos de agua.

Mediante el software ARCVIEW (ESRI 1999) se determinaron las superficies de cada cobertura del suelo en cada año. Posteriormente se compararon ambos años y se determinaron los cambios (ganancias o pérdidas) para cada tipo de cobertura. Además, se calculó la tasa de deforestación del bosque nativo utilizando una fórmula de interés compuesto (Puyravaud 2003):

$$
D=\ln \left(\frac{A_{2}}{A_{1}}\right) \times \frac{100}{t_{2}-t_{1}}
$$

Donde,

$D=$ Tasa de deforestación por año en porcentaje.

$A_{1}=$ Cobertura de bosque nativo en el tiempo 1 .

$A_{2}=$ Cobertura de bosque nativo en el tiempo 2.

$t_{1}=$ Año de evaluación inicial.

$t_{2}=$ Año de evaluación final.

Para asociar las probables causas de los cambios del bosque nativo se determinaron los flujos de cambios entre las diferentes coberturas del suelo mediante una matriz de cambios (Sandoval y Real 2005). Con esta información se determinaron los flujos específicos para coberturas de interés como bosque nativo y plantaciones de especies exóticas. De esta manera, se determinaron las coberturas del suelo que en 2003 ocupaban los sitios que en el año 1989 eran bosque nativo. Para las plantaciones de especies exóticas se determinaron las principales coberturas que ocupaban los sitios donde actualmente éstas

3 De acuerdo a la nomenclatura utilizada en el Catastro y Evaluación de los Recursos Vegetacionales de Chile (CONAF et al. 1999), la cobertura de tipo renoval corresponde a un bosque nativo secundario originado de semillas o reproducción vegetativa después de una perturbación antrópica o natural (incendio, tala rasa, derrumbe). se encuentran. Con estos resultados se produjo un mapa de cambio del bosque nativo en el cual se observan las áreas de cambio y el tipo de coberturas asociadas a estos cambios. Además, se incluyó un análisis para evaluar la relación entre la pérdida de bosque y la accesibilidad al bosque nativo. Para ello se consideró la relación entre pérdida de bosque, y variables como elevación y distancia a caminos principales, utilizando información de bases de datos del catastro de la vegetación nativa (CONAF et al. 1999).

\section{RESULTADOS}

Evaluación de la exactitud de la clasificación. Todas las áreas de entrenamiento de cada cobertura del suelo obtuvieron valores del índice de divergencia transformado mayor a 1.600, lo cual es considerado adecuado de acuerdo a ERDAS (1999). Además, la mayor parte de las áreas de entrenamiento de cada cobertura del suelo obtuvieron valores de índice de divergencia transformado cercanos a 2.000 , indicando este valor el máximo de separabilidad espectral entre pares de clases.

Se obtuvo una exactitud global de $95 \%$ y $92 \%$ para las imágenes de los años 1989 y 2003, respectivamente. La exactitud global y para cada clase se vio mejorada al agregar nueva información en ambas escenas (figura 2). En la imagen del año 1989 el aumento fue más importante, ya que la exactitud mejoró desde $83 \%$ a $95 \%$, lográndose esto al considerar las seis bandas espectrales de la imagen satelital Landsat.

En la imagen del año 2003 el aumento fue menor, mejorando desde $89 \%$ a $92 \%$ y presentando los mejores resultados de exactitud global al agregar a las primeras tres bandas espectrales, una banda de textura y otra de análisis de componentes principales. Los valores de menor exactitud se presentaron en las clases matorral y renoval, las cuales debido a su similaridad espectral producen confusiones en el algoritmo de clasificación (figura 2).

Cambios en la cobertura del suelo y el bosque nativo. En el año 1989 el área de estudio estaba dominada principalmente por las coberturas de matorral $(45 \%)$ y bosque nativo (30\%) (cuadro 1). El año 2003 la cobertura matorral siguió dominando el paisaje pero en una proporción levemente menor (43\%), el bosque nativo se vio reducido (17\%) y aumentó la participación de otras coberturas como herbáceas (17\%) y plantaciones de especies exóticas (13\%). En el período de estudio aquellas coberturas que presentaron incrementos importantes fueron: plantaciones exóticas (> 500\%) y herbáceas (> 30\%) (cuadro 1). Por otra parte, las coberturas que presentaron una mayor disminución fueron bosque nativo (44\%) y terrenos agrícolas (49\%) (cuadro 1). 


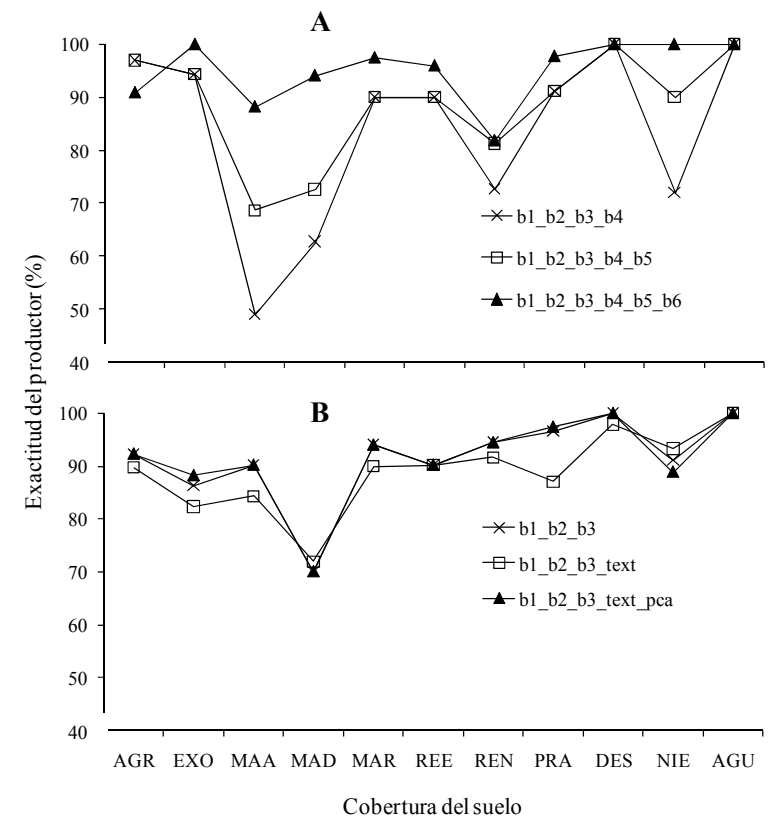

Figura 2. Exactitud de la clasificación por clase de cobertura del suelo para la imagen A) LANDSAT del año 1989 e imagen B) ASTER del año 2003, utilizando diferentes capas de información. Códigos de capas de información utilizadas: b1, b2, b3, b4, b5 y b6 = bandas espectrales de las imágenes satelitales, text $=$ banda de textura creada para la imagen Aster del año 2003, pca = banda creada mediante un análisis de componentes principales que resume la información espectral de la imagen Aster del año 2003. Códigos de cobertura del suelo: $\mathrm{AGR}=$ terrenos agrícolas, $\mathrm{EXO}=$ plantaciones de especies exóticas, MAA $=$ matorral abierto, $\mathrm{MAD}=$ matorral denso, MAR = matorral arborescente, $\mathrm{REE}=$ Renoval dominado por especies esclerófilas, REN = renoval dominado por especies de Nothofagus, PRA = praderas de uso ganadero y esteparias, DES $=$ terrenos desnudos, $\mathrm{NIE}=$ nieves, $\mathrm{AGU}=$ cuerpos de agua.

Classification accuracy per land covers class for A) the 1989 LANDSAT image and B) the 2003 ASTER image, using different layers of information. Information layer codes used: b1, b2, b3, b4, b5 and $\mathrm{b} 6=$ spectral bands of satellite images, text $=$ texture band created for 2003 Aster image, pca = band created by principal components analysis summarizing spectral information for 2003 Aster image. Land cover codes: $\mathrm{AGR}=$ agricultural lands, $\mathrm{EXO}=$ exotic species plantations, MAA $=$ open shrubland, MAD $=$ dense shrubland, MAR $=$ arborescent shrubland, REE = secondary forest dominated by sclerophyll species, REN = secondary forest dominated by Nothofagus species, PRA = cattle grazing prairies and steppes, $\mathrm{DES}=$ bare lands, NIE $=$ snow lands, $\mathrm{AGU}=$ water bodies .

La cobertura de bosque nativo se redujo desde 51.375 ha en 1989 a 28.848 ha en 2003 , lo cual correspondió a una reducción total de $44 \%$ en este período de 14 años (cuadro 1, figura 3). La tasa anual de deforestación del bosque nativo calculada para este período fue de $4,1 \%$ (1.613 ha/año).
Cuadro 1. Superficie y porcentaje de coberturas del suelo para los años 1989 y 2003 en el área de estudio.

Area and percentage of land covers between 1989 and 2003 in the study area.

\begin{tabular}{lrrrr}
\hline \multirow{2}{*}{ Cobertura del suelo } & \multicolumn{2}{c}{1989} & \multicolumn{2}{c}{2003} \\
\cline { 2 - 5 } & \multicolumn{1}{c}{ ha } & \multicolumn{1}{c}{$\%$} & \multicolumn{1}{c}{ ha } & $\%$ \\
\hline Matorral & 76.704 & 45 & 73.374 & 43 \\
Bosque nativo & 1.375 & 30 & 28.848 & 17 \\
Herbáceas & 22.282 & 13 & 29.544 & 17 \\
Plantaciones exóticas & 2.588 & 2 & 22.115 & 13 \\
Terrenos agrícolas & 6.466 & 4 & 3.270 & 2 \\
Otros & 9.716 & 6 & 11.981 & 7 \\
\hline Total & 169.131 & 100 & 169.131 & 100 \\
\hline
\end{tabular}

Un 38\% de la superficie de bosque nativo del año 1989 se mantuvo en el año 2003. La superficie de bosque nativo que se perdió durante este periodo fue reemplazada principalmente por matorral y plantaciones de exóticas, los cuales ocuparon el $29 \%$ y el $27 \%$ de la superficie que antes correspondía a bosque nativo respectivamente (cuadro 2, figura 4).

De la superficie de matorral presente en el año 2003, un 20\% correspondió a terrenos que el año 1989 eran bosque nativo, y un $68 \%$ permaneció en la misma categoría (cuadro 2). Al mismo tiempo, la superficie de plantaciones exóticas presentes el año 2003 ocupó terrenos que en el año 1989 eran principalmente bosque nativo (63\%) y matorrales (25\%), y sólo una pequeña proporción (3\%) ocupó terrenos que en el año 1989 correspondían a plantaciones de especies exóticas (cuadro 2, figura 4). Parte de la superficie de plantaciones del año 1989 fue reemplazada el año 2003 preferentemente por coberturas de matorral $(27 \%)$, herbáceas $(26 \%)$ y bosque nativo (18\%), y otra proporción se mantuvo como tal (22\%) (cuadro 2). Los terrenos agrícolas del año 1989 mostraron un patrón similar al anterior, siendo ocupados estos terrenos principalmente por matorrales $(56 \%)$ y praderas (20\%) el año 2003.

Los resultados de pérdida de bosque nativo en relación a variables de accesibilidad son mostrados en la figura 5. En el período de estudio la mayor pérdida de bosque nativo se concentró en niveles de elevación entre 600 y 1.200 m s.n.m., donde el área afectada superó el 50\% (figura 5A). En estos sectores de elevación intermedia fue donde se encontró la mayor superficie de bosque nativo en el área de estudio. Asimismo, se asociaron mayores niveles de deforestación cuando fue menor la distancia entre el bosque nativo y la red de caminos principales. Más de la mitad del área deforestada se concentró a una distancia inferior a $2,5 \mathrm{~km}$ de $\operatorname{los}$ caminos principales (figura 5B). 
A

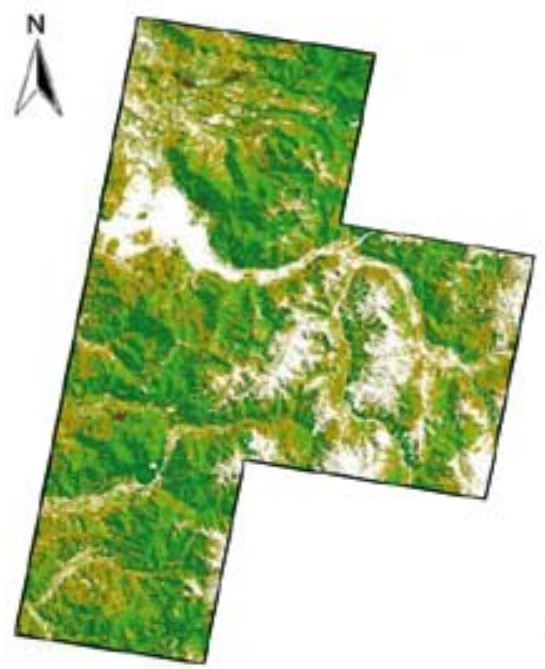

B

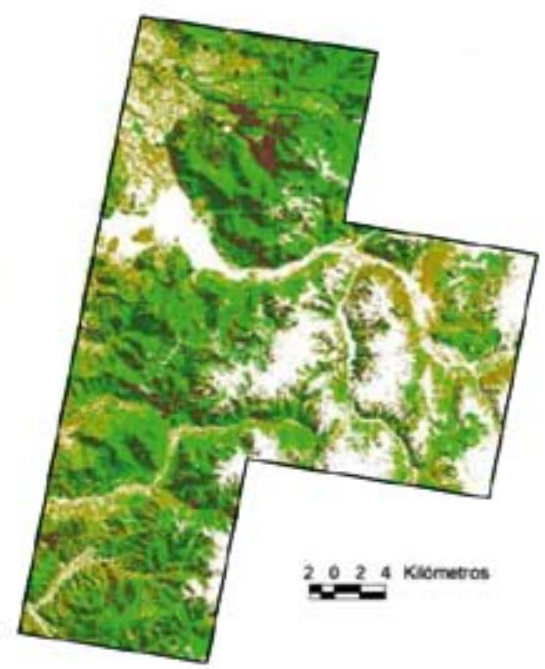

C

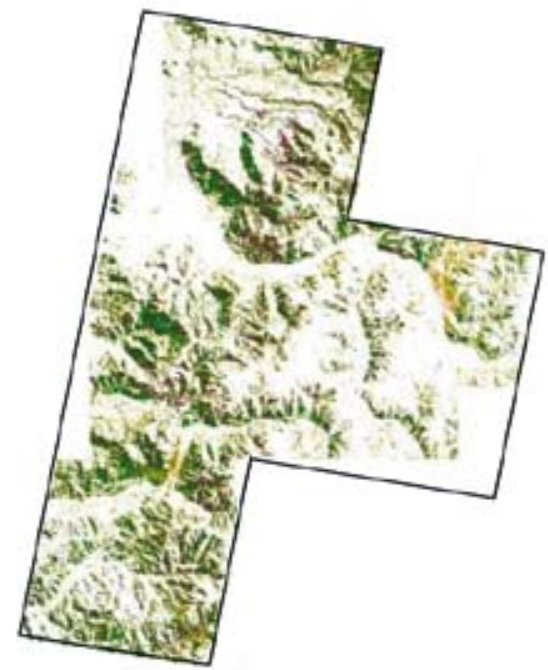

Coberturas del suelo

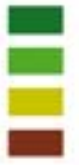

Bosque nativo

Matorral arborescente

Matorral

Plantaciones exóticas
Sin cambio y regeneración de Bosque nativo

Cambio de Bosque nativo a:

Matorral y Matorral arborescente

Plantaciones exóticas

Otras coberturas

Figura 3. Mapa de variación espacial de la cobertura de bosque nativo, matorrales y plantaciones forestales exóticas a partir de imágenes satelitales del año A) 1989 y B) 2003; y C) mapa de pérdida de bosque nativo en el área de estudio en el período 19892003. Otras coberturas incluye a terrenos agrícolas, praderas y terrenos sin vegetación.

Map of spatial variation of native forest land cover, shrublands and exotic forest plantations from satellite imagery of A) 1989 and B) 2003. C) Map of native forest loss on the study area for the period 1989-2003. Other covers include farmlands, prairies and lands without vegetation.

Cuadro 2. Matriz de cambio de la cobertura del suelo entre los años 1989 y 2003 en el área de estudio.

Matrix of land covers change between 1989 and 2003 in the study area.

\begin{tabular}{|c|c|c|c|c|c|c|c|}
\hline \multirow{2}{*}{$\begin{array}{l}\text { Cobertura del suelo } \\
\text { año } 1989 \text { (hectáreas) }\end{array}$} & \multicolumn{7}{|c|}{ Cobertura del suelo año 2003 (hectáreas) } \\
\hline & Matorral & $\begin{array}{l}\text { Bosque } \\
\text { nativo }\end{array}$ & Herbáceas & $\begin{array}{c}\text { Plantaciones } \\
\text { exóticas }\end{array}$ & $\begin{array}{l}\text { Terrenos } \\
\text { agrícolas }\end{array}$ & Otros & Total \\
\hline Matorral & 49.565 & 7.745 & 11.615 & 5.465 & 1.510 & 803 & 76.704 \\
\hline Bosque nativo & 14.723 & 19.495 & 2.434 & 13.974 & 311 & 439 & 51.375 \\
\hline Herbáceas & 4.460 & 560 & 11.856 & 947 & 573 & 3.886 & 22.282 \\
\hline Plantaciones exóticas & 707 & 470 & 666 & 581 & 7 & 157 & 2.588 \\
\hline Terrenos agrícolas & 3.622 & 394 & 1.312 & 234 & 793 & 112 & 6.466 \\
\hline Otros & 297 & 184 & 1.660 & 914 & 76 & 6.585 & 9.716 \\
\hline Total & 73.374 & 28.848 & 29.544 & 22.115 & 3.270 & 11.981 & 169.131 \\
\hline
\end{tabular}

\section{DISCUSIÓN Y CONCLUSIONES}

Uso de imágenes satelitales. En estudios multitemporales que utilizan imágenes satelitales resulta relevante alcanzar una alta exactitud, ya que de esto depende la calidad de las estimaciones que se realicen a partir de la información generada (Lillessand et al. 2004, Mather 1999). Las etapas de procesamiento y clasificación de imágenes satelitales poseen varias fuentes de error, cada una de las cuales debe ser minimizada para obtener estimaciones confiables y consistentes. Estos errores fueron minimizados mediante la aplicación de técnicas de corrección geométrica y 


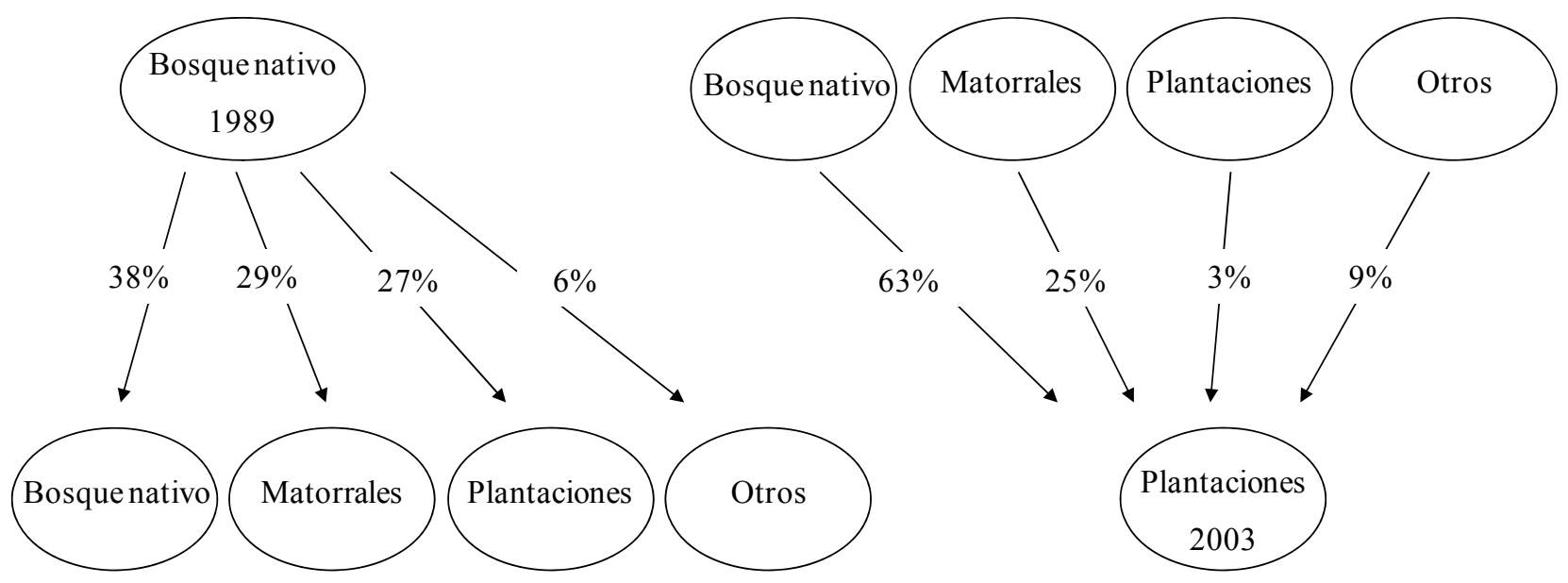

Figura 4. Trayectorias de cambio entre las diferentes coberturas del suelo entre los años 1989 y 2003. La categoría "Otros" incluye a terrenos agrícolas, praderas y terrenos sin vegetación. bare lands.

Change trajectories between the different land covers from 1989 to 2003. The category "Other" includes farmlands, prairies and
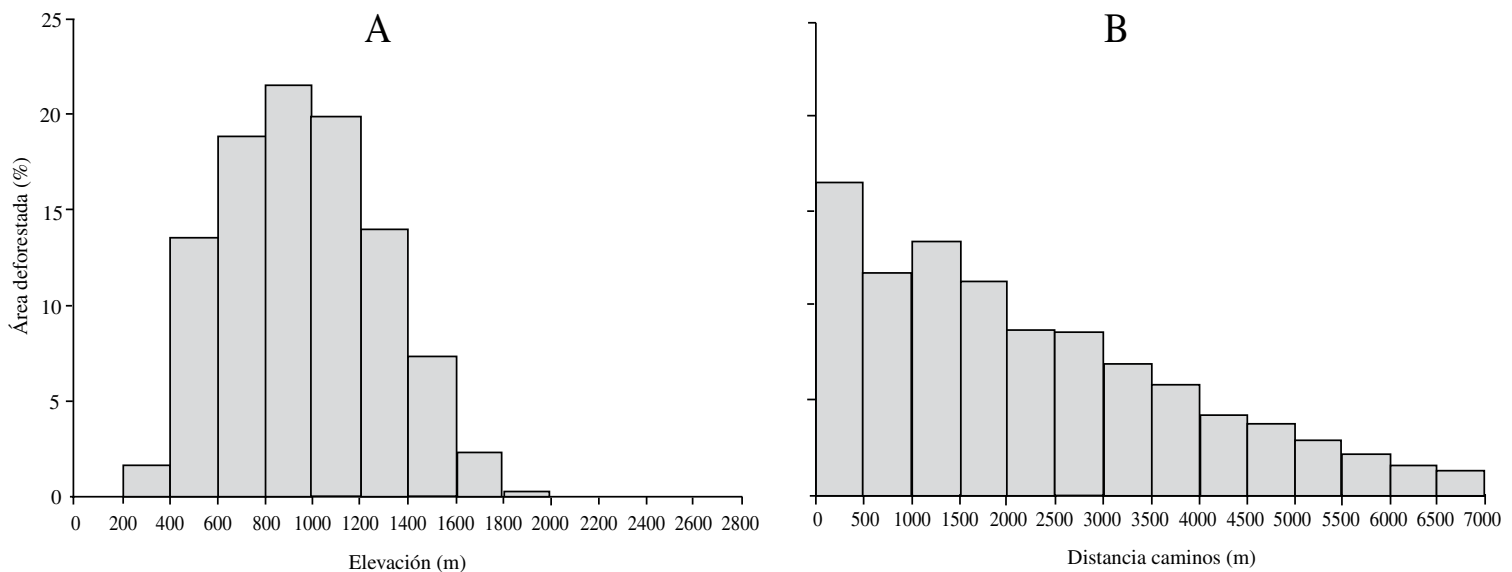

Figura 5. Porcentaje de la superficie total deforestada en el área de estudio en relación a A) elevación (m) y B) distancia a caminos (m).

Percentage of total deforested area in the study region related to A) elevation (m) and B) distance to roads (m).

atmosférica descritas en diferentes estudios que han utilizado imágenes satelitales (Liang et al. 2001, Lillesand et al. 2004). Algunos estudios también señalan que esto pudiese verse aún mejorado al aplicar una corrección topográfica, especialmente en zonas de topografía abrupta (Riaño et al. 2003). Sin embargo, otros estudios donde la topografía y nubosidad modifican los valores espectrales de las imágenes, y éstos no representan una proporción importante del área de estudio, optan por excluir las zonas de mayor sombra y nubosidad (Baldyga et al. 2007, Broadbent et al. 2008), tal como se hizo en el presente estudio.

En la etapa de clasificación de imágenes satelitales el uso de información auxiliar resultó relevante para aumentar la exactitud de la clasificación, obteniendo en ambas escenas utilizadas valores de exactitud superiores al 90\%. Similares resultados de exactitud fueron alcanzados por Armenteras et al. (2006) al utilizar información auxiliar de clima y geomorfología. Esto concuerda con Aplin (2004), quien destaca el aumento en la exactitud en la clasificación de la cobertura del suelo en estudios con imágenes satelitales al utilizar información auxiliar como datos geofísicos de elevación, temperatura, caminos y ríos. Una breve revisión de los últimos estudios publicados de cambios de la cobertura del suelo con aplicación de sensores remotos muestra valores de exactitud global similares o inferiores a los obtenidos en el presente estudio (de Barros et al. 2005, Boletta et al. 2006, Echeverría et al. 2006, Gaveau et al. 2007), lo cual ayuda a que las estimaciones de los cambios sean confiables. Otro factor incidente en la alta exactitud alcanzada fue el intenso trabajo de terreno para 
identificar las diferentes coberturas del suelo, logrando establecer más de 300 puntos de referencia distribuidos en las diferentes coberturas.

Cambio de la superficie del bosque nativo. En el período analizado destaca la alta tasa de deforestación $(4,1 \%)$ en comparación a otros estudios de la zona. En esta misma área, entre los años 1987 y 1989, Olivares (1999) detectó una tasa de deforestación de 1,4\% utilizando fotografías aéreas y una fórmula de tasa de deforestación absoluta. Un estudio reciente de la zona costera de la Región del Maule, utilizando imágenes satelitales, reportó una tasa de deforestación de 3,6\% entre los años 1990 y 2000 (Echeverría et al. 2006).

Al realizar una comparación con estudios recientes que han cuantificado la tasa de deforestación en ecosistemas de otras partes del mundo quedan de manifiesto los altos niveles de deforestación reportados en este estudio (cuadro 3). Tasas de deforestación similares o mayores sólo se han reportado en bosques caducifolios de la zona semiárida del Chaco en Argentina (Boletta et al. 2006) y en bosques tropicales de Chiapas en México (Cayuela et al. 2006) (cuadro 3). Sin embargo, se debe tener precaución en estas comparaciones, ya que pueden existir diferencias en el tipo de información utilizada y especialmente en los criterios de clasificación de la vegetación.

Causas de los cambios del bosque nativo. Por largo tiempo los bosques del área de estudio han sido seriamente deforestados y degradados debido a la extracción de árboles para habilitación de terrenos agrícolas y para producción de leña para combustible (San Martín y Donoso 1995). Las causas de la alta tasa de deforestación encontrada en este estudio también podrían asociarse al alto consumo de maderas nativas para uso industrial, como ha sido reportado en estudios anteriores (Lara et al. 2002). Sin embargo, el consumo industrial de maderas nativas, a nivel nacional, disminuyó desde 2,3 millones de metros cúbicos en 1990 a sólo 0,6 millones de metros cúbicos en el año 2003 (INFOR 2004). Además, a partir del año 1995, y específicamente en la Región del Maule, no se registra producción de astillas de madera nativa (INFOR 2005), lo cual podría indicar que en este período ha existido una menor presión sobre el bosque nativo a partir de estas fuentes. De esta manera, los altos niveles de deforestación deberían estar asociados a otras causas.

Los resultados de este estudio indican que la conversión del bosque nativo en el período de estudio ha sido principalmente hacia matorrales $(29 \%)$ y plantaciones forestales de especies exóticas (27\%) (principalmente $P$. radiata). Las causas asociadas a la conversión hacia matorrales pueden deberse en primer término al desarrollo de prácticas de floreo, las cuales se caracterizan por la extracción de los árboles de mejor calidad. Este tipo de actividades produce una alta degradación del bosque, dando origen a estas formaciones de tipo matorral (Donoso 1994, San Martín y Donoso 1995). Otra causa de este tipo de conversión se asocia a la extracción de leña, la cual es utilizada como medio de subsistencia por muchos pequeños propietarios en varias zonas del país (Reyes 2000). El uso del bosque nativo como combustible para leña también ha producido una gran degradación de este recurso (Olivares 1999) dando origen a formaciones de tipo matorral. En particular, esta causa asociada a la deforestación podría ser de las más relevantes, ya que de acuerdo a datos de INFOR (2004) ha habido un aumento sostenido en el consumo de este tipo de combustible en los últimos años. Tanto las prácticas de floreo como la extracción de leña se ven favorecidas por una mayor accesibilidad, lo cual coincide con los resultados encontrados, donde los mayores niveles de deforestación se asocian a elevaciones intermedias y una menor distancia a los caminos (figura 5).

La conversión del bosque nativo hacia plantaciones exóticas es la segunda causa de mayor relevancia para el bosque nativo, lo cual también se ve reflejado debido a que una gran proporción de las actuales plantaciones están establecidas sobre terrenos que anteriormente correspondían a bosque nativo (63\%) (cuadro 2, figura 4). En general, en el área de estudio las plantaciones exóticas están establecidas sobre terrenos de buena accesibilidad, lo cual

Cuadro 3. Tasas anuales promedio de deforestación estimadas por estudios recientes en diferentes regiones del mundo.

Recent estimates of deforestation rates in different regions of the world.

\begin{tabular}{|c|c|c|c|}
\hline Autor & País & Período estudio & Tasa anual de deforestación (\%) \\
\hline Echeverría et al. 2008 & Chile* & 1985-1999 & 0,78 \\
\hline Gaveau et al. 2007 & Sumatra & $1972-2002$ & $2,86 * * *$ \\
\hline Armenteras et al. 2006 & Colombia & $1985-2001$ & 3,70 \\
\hline Boletta et al. 2006 & Argentina & 1992-1999 & 5,00 \\
\hline Cayuela et al. 2006 & México & $1990-2000$ & 4,80 \\
\hline Echeverría et al. 2006 & Chile** & $1975-2000$ & 4,50 \\
\hline Ewers et al. 2006 & Nueva Zelanda & $1997-2002$ & 0,01 \\
\hline de Barros et al. 2005 & Brasil & 1984-2002 & $2,90 * * *$ \\
\hline
\end{tabular}

* Sur de la Región de Los Lagos. ** Costa de la Región del Maule. *** Tasa de deforestación calculada linealmente y no utilizando fórmula de interés de compuesto. 
también es concordante respecto a los mayores niveles de deforestación en relación a elevación y distancia a caminos (figura 5). En esta misma área, estudios de épocas previas reportaron que la principal disminución de la superficie de bosque nativo se asociaba a la sustitución por especies exóticas (Olivares 1999, CONAF y UACh 2000). Esto se explica por el crecimiento que ha experimentado la industria después de la aplicación de la ley de incentivo a la forestación en el año 1976, y por la creciente demanda de productos derivados de la madera y pulpa (Lara et al. 2002). Sin embargo, algunos antecedentes a nivel nacional indican que la sustitución de bosque nativo por plantaciones exóticas podría estar disminuyendo, lo que provocaría un descenso en la tasa de forestación anual (INFOR 2005). El mismo efecto podría ser atribuible al comienzo (a partir del año 1997) de un proceso gradual de certificación de las empresas forestales a través de diferentes sistemas, lo cual ha dado origen a un compromiso de algunas empresas con el manejo sustentable de los recursos forestales del país (Lara et al. 2002). Esto último no elimina la existencia de un problema, ya que esta causa continúa siendo una de las más relevantes en la deforestación del bosque nativo. De esta manera, el bosque nativo continúa transformándose gradualmente en matorrales y plantaciones exóticas, dando origen a bosques de baja calidad, e implicando procesos de fragmentación que tienen diversos efectos negativos sobre los ecosistemas.

Implicancias para la conservación y el manejo de los recursos naturales. Las altas tasas de deforestación tienen un impacto negativo sobre la biodiversidad, ya que este proceso conduce a la fragmentación del paisaje mediante la pérdida de bosque, reducción del tamaño, y aumento del aislamiento de los fragmentos remanentes (Echeverría et al. 2006), lo cual a su vez afecta los procesos ecológicos (Armenteras et al. 2006).

Los altos niveles de deforestación encontrados pueden representar una seria amenaza para estos ecosistemas, los cuales están presentes en una zona considerada como una de las más importantes a nivel mundial en términos de biodiversidad (Arroyo et al. 1996, Myers et al. 2000, Smith-Ramírez 2004). A su vez, de acuerdo a la Iniciativa Global 200 de la WWF y el Banco Mundial, estas áreas se encuentran entre las más amenazadas del mundo (Dinerstein et al. 1995).

La deforestación y fragmentación pueden producir cambios importantes en el hábitat de las especies, en particular para las especies amenazadas (Altamirano et al. 2007). En el área de estudio es posible encontrar algunas especies amenazadas como N. glauca, A. chilensis, B. berteroana y $C$. mucronata. Este tipo de especies tienen una distribución restringida y poseen hábitats altamente específicos (Hechenleitner et al. 2005), pudiendo ver afectada su persistencia en el tiempo debido a cambios en la luminosidad, temperatura y humedad producto de los altos niveles de deforestación y fragmentación (Altamirano et al. 2007).
La deforestación también puede afectar negativamente la regulación de los caudales y la producción hídrica. Los bosques nativos, en comparación a otras coberturas del suelo, tienen un efecto regulador que permite un abastecimiento constante de agua, evitando inundaciones de invierno y permitiendo disponibilidad de este recurso en los meses más secos del verano (Otero et al. 1994, Vertessy et al. 2001, Oyarzún et al. 2004). Esta situación es muy relevante en la zona de estudio, debido a que es la productora de casi un $50 \%$ del total de energía eléctrica que se genera en el país (Lara et al. 2003). En otras latitudes se han detectado problemas sobre la industria hidroeléctrica producto de la deforestación. Por ejemplo, estudios en países de Europa y América Central han reportado que la deforestación está afectando la producción de agua y vida útil de las represas hidroeléctricas, esto último debido a los altos niveles de sedimentación que son producidos por la pérdida de cobertura forestal (Haigh et al. 2004, Mwendera 2006). Problemas de abastecimiento de agua pueden tener serias repercusiones de tipo social, ya que la mayor parte de las comunidades aledañas a los bosques depende del recurso hídrico para su propia subsistencia y regadío de cultivos, lo cual acentúa los problemas de pobreza y migración rural (Lara et al. 2003).

La dinámica de cambios que se produce en el área de estudio también ve afectada de manera importante a otras coberturas del suelo como las plantaciones de especies exóticas y terrenos agrícolas (cuadro 2). La conversión de estos terrenos principalmente en matorrales sugiere el abandono de estos terrenos después de la cosecha y su posterior colonización por especies pioneras, lo cual origina estos estados iniciales de sucesión. Donoso (1994) señala que estos procesos de recuperación en los bosques esclerófilos son más rápidos en sectores húmedos que en sectores secos, y que el proceso dinámico de sucesión es distinto en ambos casos. La ocurrencia de este proceso representa una oportunidad para llevar a cabo estrategias de restauración y recuperación de áreas degradadas, lo cual a su vez requiere de un estudio de las estrategias que utilizan las especies para colonizar las diferentes condiciones de sitio que se han visto alteradas.

Medidas de mitigación. Dado que las actuales prácticas de extracción de madera, principalmente floreo y uso como combustible, corresponderían a las principales causas atribuibles a la alta tasa de deforestación, se hace imperioso contar con un sistema que incentive prácticas de manejo adecuado. Recientemente se promulgó la nueva ley de fomento y recuperación del bosque nativo, la cual incorpora incentivos económicos para los propietarios de bosque nativo (Lara et al. 2003). El monto y focalización de estos incentivos será vital en el potencial efecto que se desea con esta ley. Debido a esto, y bajo un usual escenario de recursos limitados, los incentivos debiesen focalizarse considerando los siguientes elementos: niveles de deforestación, tenencia de la propiedad, presencia de 
especies amenazadas y nivel de disponibilidad de agua desde las cuencas. Esta priorización de áreas podría ayudar a proteger las cuencas aledañas a los embalses de la zona, los cuales requieren una urgente protección (Lara et al. 2003). De esta manera, los incentivos no sólo contribuirían a la conservación de los recursos naturales, sino también incentivarán actividades económicas que beneficiarán a las comunidades aledañas.

Debido a que los actuales incentivos también consideran apoyo para áreas de conservación y recuperación, estos deberían priorizar el mejoramiento de la conectividad de las actuales áreas protegidas, lo cual aumenta las posibilidades de sobrevivencia de las especies amenazadas (Piessens et al. 2005). En el área de estudio se encuentran dos áreas protegidas (Reserva Nacional Altos de Lircay y Los Bellotos), distanciadas en aproximadamente $20 \mathrm{~km}$ y ubicadas en la precordillera de Los Andes. Sin embargo, estas áreas se encuentran totalmente desconectadas con las áreas ubicadas hacia la zona occidental, distanciándose por más de $100 \mathrm{~km}$ con el área protegida más cercana (CONAF et al. 1999).

Las plantaciones de especies exóticas presentaron un incremento importante en el período de estudio, siendo también una de las principales fuentes de reemplazo del bosque nativo. En este caso particular es necesario contar con un sistema de fiscalización eficiente, el cual monitoree de forma permanente los predios sujetos a planes de manejo. Las actuales tecnologías permiten desarrollar programas de monitoreo eficaces para actividades de manejo forestal (Lutz et al. 2008), lo cual puede ser una medida que ayude a controlar el avance de las plantaciones exóticas sobre terrenos con bosque nativo. Investigaciones futuras sobre el cambio de la cobertura del suelo, y en particular el bosque nativo, requieren la utilización de modelos espacialmente explícitos que incorporen variables sociales para identificar las fuentes (grandes empresas o pequeños y medianos propietarios de bosques) y causas directas del reemplazo del bosque nativo por otras coberturas del suelo.

\section{AGRADECIMIENTOS}

Los autores agradecen el financiamiento al proyecto MECESUP AUS 0103 y al Núcleo Científico FORECOS P04-065-F (Servicios ecosistémicos del bosque nativo a sistemas acuáticos bajo fluctuaciones climáticas) financiado por la iniciativa Científica Milenio de MIDEPLAN. También agradecemos al Sr. Andrés Rivera del Centro de Estudios Científicos de Valdivia (CECS) por facilitar una imagen satelital ASTER.

\section{REFERENCIAS}

Altamirano A, C Echeverría, A Lara. 2007. Efecto de la fragmentación forestal sobre la estructura vegetacional de las poblaciones amenazadas de Legrandia concinna (Myrtaceae) del centro-sur de Chile. Revista Chilena de Historia Natural 80: 27-42.

Alves DS. 2002. Space-time dynamics of deforestation in Brazilian Amazonia. International Journal of Remote Sensing 23(14): 2903-2908.

Aplin P. 2004. Remote sensing: land cover. Progress in Physical Geography 28(2): 283-293.

Armenteras D, G Rudas, N Rodríguez, S Sua, M Romero. 2006. Patterns and causes of deforestation in the Colombian Amazon. Ecological Indicators 6: 353-368.

Armesto JJ, R Rozzi, C Smith-Ramírez, MK Arroyo. 1998. Conservation targets in South American temperate forests. Science 282: 1271-1272.

Arroyo MK, L Cavieres, A Peñaloza, M Riveros, AM Faggi. 1996. Relaciones fitogeográficas y patrones regionales de riqueza de especies en la flora del bosque lluvioso templado de Sudamérica. In Armesto J, C Villagrán, MTK Arroyo (eds). Ecología de los Bosques Nativos de Chile. Santiago, Chile. Editorial Universitaria. p. 71-99.

Baldyga T, SN Miller, KL Driese, C Maina Fichaba. 2007. Assessing land cover change in Kenya's Mau Forest region using remotely sensed data. African Journal of Ecology 46: $46-54$

Boletta PE, AC Ravelo, AM Planchuelo, M Grillo. 2006. Assessing deforestation in the Argentine Chaco. Forest Ecology and Management 228: 108-114.

Broadbent EN, GP Asner, M Keller, DE Knapp, PJC Oliveira, JN Silva. 2008. Forest fragmentation and edge effects from deforestation and selective logging in the Brazilian Amazon. Biological Conservation 141: 1745 -1757.

Bustamante R, C Castor. 1998. The decline of an endangered temperate ecosystem: the ruil (Nothofagus alessandrii) forest in central Chile. Biodiversity and Conservation 7: 1607-1626.

Cayuela L, J M Rey Benayas, C Echeverría. 2006. Clearance and fragmentation of tropical montane forests in the Highlands of Chiapas, Mexico (1975-2000). Forest Ecology and Management 226: 208-218.

CONAF (Corporación Nacional Forestal, CL), UACh (Universidad Austral de Chile, CL). 2000. Monitoreo y Actualización de la Información de uso actual del suelo en la VII Región. Proyecto CONAF-UACH. Informe final. $58 \mathrm{p}$.

CONAF (Corporación Nacional Forestal, CL), CONAMA (Comisión Nacional del Medio Ambiente, CL), BIRF (Banco Internacional de Reconstrucción y Fomento, USA), Universidad Austral de Chile, Pontificia Universidad Católica de Chile, Universidad Católica de Temuco. 1999. Catastro y evaluación de los recursos vegetacionales nativos de Chile. Santiago, Chile. CONAF, 88 p. (Informe nacional con variables ambientales).

Coppin P, I Jonckheere, K Nackaerts, B Muys. 2004. Digital change detection methods in ecosystem monitoring: a review. International Journal of Remote Sensing 25(9): 1565-1596.

D’Almeida C, CJ Vörösmarty, JA Marengo, GC Hurtt, SL Dingman, BD Keim. 2006. A water balance model to study the hydrological response to different scenarios of deforestation in Amazonia. Journal of Hydrology 331: 125-136.

De Barros Ferraz SF, CA Vettorazzi, DM Theobald, MV Ramos Ballester. 2005. Landscape dynamics of Amazonian 
deforestation between 1984 and 2002 in central Rondônia, Brazil: assessment and future scenarios. Forest Ecology and Management 204: 67-83.

Di Castri F, ER Hajek (1976) Bioclimatología de Chile. Santiago, Chile. Imprenta-Editorial de la Universidad Católica de Chile. 129 p.

Dinerstein E, DM Olson, DJ Graham, AL Webster, SA Primm, MP Bookbinder. 1995. Una evaluación del estado de conservación de las ecorregiones terrestres de América Latina y el Caribe. Washington, DC. USA. Banco Mundial/WWF. $129 \mathrm{p}$.

Donoso C. 1994. Bosques templados de Chile y Argentina. Variación, estructura y dinámica. Segunda Edición. Santiago, Chile. Editorial Universitaria. 484 p.

Donoso C, A Lara 1995. Utilización de los bosques nativos en Chile: pasado, presente y futuro. In Armesto J, C Villagrán, M Arroyo eds. Ecología de los bosques nativos de Chile. Santiago, Chile. Editorial Universitaria. p. 363-387.

Echeverría C, D Coomes, J Salas, JM Rey-Benayas, A Lara, A Newton. 2006. Rapid deforestation and fragmentation of Chilean temperate forests. Biological Conservation 130: 481-494.

Echeverría C, D Coomes, M Hall, AC Newton. 2008. Spatially explicit models to analyze forest loss and fragmentation between 1976 and 2020 in southern Chile. Ecological Modelling 212: 439-449.

Ewers R, A Kliskey, S Walker, D Rutledge, J Harding, R Didham. 2006. Past and future trajectories of forest loss in New Zealand. Biological Conservation 133: 312 -325.

ERDAS. 1999. Erdas Field Guide. Fifth edition, revised and expanded. Atlanta Georgia, USA. Erdas Inc. 698 p.

ESRI. 1999. Environmental Systems Research Institute, Inc., Redlands, CA, USA.

Etter A, C Mcalpine, K Wilson, S Phinn, H Possingham. 2006. Regional patterns of agricultural land use and deforestation in Colombia. Agriculture, Ecosystems and Environment 114: 369-386.

Gaveau DLA, H Wandono, F Setiabudi. 2007. Three decades of deforestation in southwest Sumatra: Have protected areas halted forest loss and logging, and promoted re-growth? Biological Conservation 134(4): 495-504.

Haigh MJ, L Jansky, J Hellin. 2004. Headwater deforestation: a challenge for environmental management. Global Environmental Change 14: 51-61.

Hechenleitner P, M Gardner, P Thomas, C Echeverría, B Escobar, P Brownless, C Martínez. 2005. Plantas amenazadas del centro-sur de Chile. Distribución, conservación y propagación. Valdivia, Chile. Trama Impresores. 188 p.

INFOR (Instituto Forestal, CL). 2004. Estadísticas Forestales 2003. Santiago, Chile. 81 p. (Boletín Estadístico 95).

INFOR (Instituto Forestal, CL). 2005. Estadísticas Forestales Región del Maule, 2004. Santiago, Chile. 81 p. (Boletín estadístico 104).

Kerr J, M Ostrovsky. 2003. From space to species: ecological applications for remote sensing. Trends in Ecology and Evolution 18(6): 299-305.

Lara A, C Donoso, JC Aravena. 1995. La conservación del bosque nativo en Chile: Problemas y desafíos. In Armesto J, C Villagrán, M Arroyo eds. Ecología de los bosques nativos de Chile. Santiago, Chile. Editorial Universitaria. p. $335-362$.
Lara A, C Echeverría, R Reyes. 2002. Bosques Nativos. In Instituto de Asuntos Públicos, Universidad de Chile eds. Informe País. Estado del Medioambiente en Chile. Santiago, Chile. p. 127-160

Lara A, L Araya, J Capella, M Fierro, A Cavieres. 1989. Evaluación de la destrucción y disponibilidad de los recursos forestales nativos en la VII y VIII Región. Santiago, Chile. 22 p. (Informe Técnico CODEFF).

Lara A, D Soto, J Armesto, P Donoso, C Wernli, L Nahuelhual, F Squeo eds. 2003. Componentes científicos clave para una política nacional sobre usos, servicios y conservación de los bosques nativos chilenos. Libro resultante de la reunión científica sobre bosques nativos realizada en Valdivia, los días 17-18 julio de 2003. Valdivia, Chile. Universidad Austral de Chile. 134 p. (Iniciativa Científica Milenio de Mideplan).

Laurance WF, AKM Albernaz, G Schroth, PM Fearnside, S Bergen, EM Venticinque, C da Costa. 2002. Predictors of deforestation in the Brazilian Amazon. Journal of Biogeography 29(5): 737-748.

Liang S, H Fang, M Chen. 2001. Atmospheric Correction of Landsat ETM+ Land Surface Imagery-Part I: Methods. IEEE Transactions on Geoscience and Remote Sensing 39(11): 2490-2497.

Lillesand T, R Kiefer, J Chipman. 2004. Remote Sensing and image interpretation. New York, USA. John Wiley \& Sons, Fifth edition. 763 p.

Lutz DA, RA Washington-Allen, HH Shugart. 2008. Remote sensing of boreal forest biophysical and inventory parameters: a review. Canadian Journal of Remote Sensing 34: 286-313.

Mainville N, J Webb, M Lucotte, R Davidson, O Betancourt, E Cueva, D Mergler. 2006. Decrease of soil fertility and release of mercury following deforestation in the Andean Amazon, Napo River Valley, Ecuador. Science of the Total Environment 368: 88-98.

Mather P. 1999. Computer Processing of Remotely-Sensed images. An introduction. Chichester, England. John Wiley \& Sons, Second Edition. 292 p.

Myers N, RA Mittermeler, CG Mittermeler, GAB da Fonseca, J Kent. 2000. Biodiversity hotspots for conservation priorities. Nature 403: 853-858.

Mwendera EJ. 2006. Available water for hydropower generation in Swaziland. Physics and Chemistry of the Earth 31: 952-959.

Olivares P. 1999. Sustitución de bosque nativo por otros usos del suelo en dos sectores de la precordillera andina de la VII Región, entre los años 1987 y 1996. Tesis de grado Ingeniería Forestal.Valdivia, Chile. Facultad de Ciencias Forestales, Universidad Austral de Chile. 81 p.

Otero L, A Contreras, A Barrales. 1994. Efectos ambientales de diferentes tipos de cortas en bosque nativo. El caso de las cortas de protección en fajas. Ciencia e Investigación Forestal 8(1): 87-118.

Oyarzún C, L Nahuelhual, D Núñez. 2004. Los servicios ecosistémicos del bosque templado lluvioso: producción de agua y su valoración económica. Revista Ambiente y Desarrollo 20(3) - 21(1): 88-95

Pezoa L. 2003. Recopilación y análisis de la variación de las temperaturas (período 1965-2001) y las precipitaciones (período 1931-2001) a partir de la información de estaciones meteorológicas de Chile entre $\operatorname{los} 33^{\circ}$ y $53^{\circ}$ de latitud sur. 
Tesis Ingeniero Forestal. Valdivia, Chile. Facultad de Ciencias Forestales, Universidad Austral de Chile. 99 p.

Piessens K, O Honnay, M Hermy. 2005. The role of fragment area and isolation in the conservation of heathland species. Biological Conservation 122: 61-69.

Puyravaud JP. 2003. Standardizing the calculation of the annual rate of deforestation. Forest Ecology and Management 177: 593-596.

Reyes R. 2000. Caracterización de los sistemas de producción y comercialización de leña para la ciudad de Puerto Montt, X Región. Tesis Ingeniero Forestal. Facultad de Ciencias Forestales, Universidad de Chile, Santiago, Chile.

Riaño D, E Chuvieco, J Salas, I Aguado. 2003. Assessment of Different Topographic Corrections in Landsat-TM Data for Mapping Vegetation Types. IEEE Transactions on Geoscience and Remote Sensing 41(5): 1056-1061.

Sandoval V, P Real. 2005. Modelamiento y prognosis estadística y cartográfica del cambio en el uso de la tierra. Bosque 26(1): 55-63.

San Martín J, C Donoso. 1995. Estructura florística e impacto antrópico en el bosque maulino de Chile. In Armesto J, C Villagrán, M Arroyo eds. Ecología de los bosques nativos de Chile. Santiago, Chile. Editorial Universitaria. p. 153-168.

Schlatter JE, V Gerding, J Adriazola. 1997. Sistema de Ordenamiento de la Tierra: Herramienta para la planificación forestal aplicada a las Regiones VII, VIII y IX. Valdivia, Chile. Facultad de Ciencias Forestales, Universidad Austral de Chile, Segunda edición. 81 p.

Sheeran K. 2006. Forest conservation in the Philippines: A costeffective approach to mitigating climate change? Ecological Economics 58: 338- 349.

Smith-Ramírez C. 2004. The Chilean coastal range: a vanishing center of biodiversity and endemism in South American temperate rainforests. Biodiversity and Conservation 13: 373-393.

Sun G, SG Mcnulty, J Lu, DM Amatya, Y Liang, RK Kolka. 2005. Regional annual water yield from forest lands and its response to potential deforestation across the southeastern United States. Journal of Hydrology 308: 258-268.

Vertessy RA, FGR Watson, SK O'Sullivan. 2001. Factors determining relations between stand age and catchment water balance in mountain ash forests. Forest Ecology and Management 143(1-3): 13-26.

Zimmermann B, H Elsenbeer, JM de Moraes. 2006. The influence of land-use changes on soil hydraulic properties: Implications for runoff generation. Forest Ecology and Management 222: 29-38.

Recibido: 23.02.09

Aceptado:17.11.09 